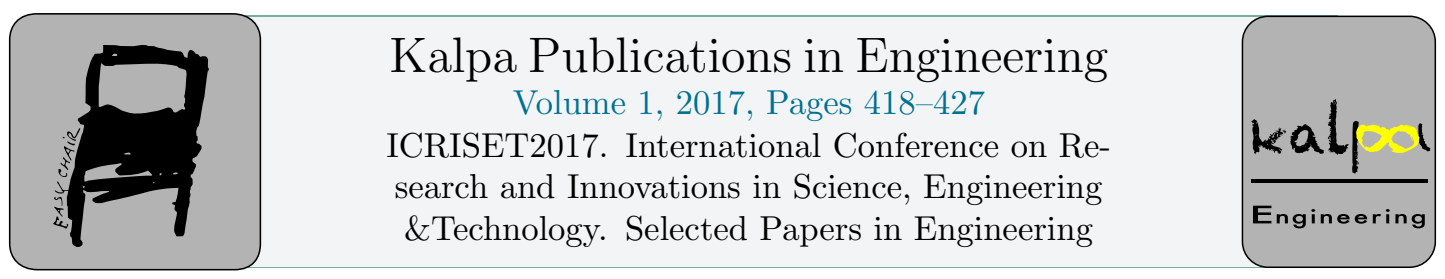

\title{
Robust Gait Recognition Technique Using Neural Network
}

\author{
Ripal Patel ${ }^{1}$ (Asst. Professor), Vivek Tank ${ }^{1}$, \\ Jinish Brahmbhatt ${ }^{1}$, Sanchit Puranik ${ }^{1}$ and Khushboo Desai ${ }^{1}$ \\ ${ }^{1}$ B.V.M. College of Engineering \\ ripal.patel@bvmengineering.ac.in, vivektnk@gmail.com
}

\begin{abstract}
With the increase in the number of thefts, robberies and encroaching in the world, the existing security system is not sufficient. Hence to circumvent this problem the demand of biometric systems is increased, as they provide more dependable and effective means of identity confirmation. One such biometric security that has seen uproar in the recent years is the gait identification. Gait recognition targets fundamentally to address this problem by recognizing people based on the way they walk. First of all, silhouette of the persons is extracted. Moreover, step size of the person is considered as a unique feature for representing gait and effectively classifies the person based on gait. Finally, features are feed to neural network for classification. The proposed approach gives better accuracy.
\end{abstract}

\section{Introduction}

The systematic study of human locomotion or animal movement by using the eye and brain of the observers, in combination with instrumentation for measuring the body movements, activities of the muscles or body mechanics is known as Gait Analysis. The walking movement that is achieved by the motion of the human limbs is known as human gait. The human gait is defined as bipedal (i.e. walking on 2 feet) and biphasic which is the result of sinuous movements along with forward progression of the different body parts. The study involves quantification (i.e. analysis of measurable parameters of gait) and interpretation (i.e. drawing various conclusions about the human) from its gait pattern.

Gait patterns are characterized by certain parameters like overall velocity, forces, and the difference in the limb movement patterns and changes in the contact with the surface. Therefore, the human gait 
can be distinguished from others using these unique features. The two types of gait are Natural Gait and Abnormal Gait.

Natural Gait - As the name suggests these occur in humans naturally. They aren't the result of any specialized training or injury in the lower parts of the body. These include sprint, run, skip, jog and walk.

Abnormal Gait - These do not occur naturally in humans; they are a result of specialized training or injury in the lower section of the body.

\section{Literature survey}

The proposed method presented (Ross Cutler, 2008) talks about the type of method for identifying an ambulatory subject based on video analysis of the subject's gait. In this method they take a sequence of images that collectively captures the gait of the subject, determining a collection of features from the sequence of images representative of the gait, and identifying the subject based on gait. The subject is identified on the basis of the features of cadence, stride length and height parameters. Gait recognition refers to the task of automatically extracting visual cues that characterize the motion of a walking person and using the clues to potentially identify the person. Gait is an interactive biometric for automated recognition, it has the ability to determine this biometric at a distance, i.e. without the need to interact with the subject, or even the cooperation of the subject. They have also looked into the troubles like due to environmental conditions the quality of captured images may degrade, the video might be of low resolution, and the subject's apparel may obscure the gait behavior. This factors may affect the gait analysis results to great extent.

An idea, behind the invention of the method proposed (Yu Zhong, 2015)is that we can identify an individual depending on a person's gait. We need to know the speed of a person which data is received from an accelerometer carried by a person which is embedded in the person's mobile phone. The acceleration data corresponds to the gait of the person. A gait dynamics image [GDI] is calculated based on the rotational invariant values. The GDI as mentioned above is matched to the set of predetermined GDIs to identify the person. This matching can be done using correlation methods. The two applications for this invention are: Firstly, the test gait features are compared with pre-stored gait features to identify that person is known to system or not. Secondly, for authentication where the comparison between the enrolled gait data and the gait of known person is done to validate the identity. This method has the accuracy of about approximately $50 \%$. The technique of accelerometer based gait recognition techniques work fairly as they are under constrained conditions, their performance degrades in real world applications as there are variations in sensor placement, apparel and performed activities persist.

The Principle component analysis (PCA) is one of the methods proposed (Nisha Shroti and Prof. Anil Khandekar, 2015) used for the gait recognition. It performs better in the recognition but requires higher computation time. The gait is the dynamic feature in the human which is being proved as strong recognition abilities. This feature does not require any type of cooperation from the observed individual, and by using non-expensive cameras which is one of the benefits that has been drawing 
enormous attention to gait biometric process. This paper shows a new gait recognition algorithm based on KPCA. All experiments were carried out using the silhouette dataset A of CASIA gait database system.

Another method, proposed (Liang Wang \& Tieniu Tan, 2003) uses spatio-temporal silhouette analysis. Conventional and simple background subtraction technique had been used in this approach. Spatio-temporal analysis of silhouette considers the change in silhouette with time. Later on it is feed to Principle component analysis system to describe more information in term of small and compact principle component. Jiwen Lu and Erhu Zhang [5] have proposed a simple method for gait identification by using human silhouettes for Independent Component Analysis (ICA) and multiple feature representation. The problems of principle component analysis are overcome by ICA. The author used simple background subtraction method to achieve silhouette in terms of binary image. Then ICA is applied to the silhouettes for reducing computational complexities. Finally, the features from ICA are provided to the nearest neighbor classifier for labeling the object.

In the method proposed(Alvaro Muro-de-la-Herran, Begonya Garcia-Zapirain,and Amaia MendezZorrilla, 2014) they research the system using an image processing method for analysis or recognition of gait of a moving object which can be either human or non-human being. This paper mainly targets three methods (1) Image processing (2) floor sensor and (3) sensors placed on the body. Here the $3^{\text {rd }}$ method is the most promising method in the case of efficiency, precision, conformability, usability or transportability. This project also can be used in the field of medical. Through which we can tell the quality of the life of any person. By evaluating and monitoring the gait we will enable to find the disease and its effects and help to find the best treatment. This project can be used in the field of (a) neurological diseases, (b) systemic diseases such as cardiopathies, (c) alterations in de-ambulation dynamic, (d) diseases caused by aging. In this paper, the system is divided into two different approaches based on the non-wearable sensor (NWS) and another is on wearable sensors (WS). The NWS systems require the specific facilities where sensors are placed at the specific places to analyses or monitor or capture the gait while the person walks on the walking path. WS systems make it possible to analyzing or monitoring the gait anywhere means not only in walkway or offices but anyplace at any time and we can get the result sitting in our office. There is also a third approach which is hybrid of NWS and WS systems.

In this proposed approach (Zhaoxiang Zhang, Maodi Hu and Yunhong Wang, 2011) mainly they are analyzing the biometric gait by which they are acquiring the biometric information like identity, gender, ethnicity, and age from the persons walking. With reference to another biometrics, gait can be measured from distance. Gait is hard to replicate as compared to another biometrics of humans. From all the biometric identification gait is most appropriate and efficient recognition and analysis technique. E.g.- face recognition is not always suitable for surrounding atmosphere like sometimes the angle of the face is different, or face is too far away or too close to the camera, so the camera could not get the full face for the recognition. While gait is behavioral biometric. Which not only depends on the appearance of a particular person but it also includes the height of the person, length of the leg of the person, shoulder width of the person, and most important dynamics of individual walking. Gait 
cycle can be divided in some parameters for the gait recognition like movements of joint and muscle which also includes body weight, limb length, and joint velocity and bone structure.

\section{Proposed Method}

The block diagram of proposed gait recognition algorithm is shown in figure 1. Input video sequence is the first block in our system, which provides the necessary picture frames for the feature extraction.

\subsection{Feature Extraction}

Feature extraction uses an algorithm which will help the system recognize the gait faster and easier. Feature extraction is used when a large amount of data is fed to the input of any system. The large data may contain a lot of redundant information which needs to be converted into reduced data set. In this method, we found out the step length of the various people.

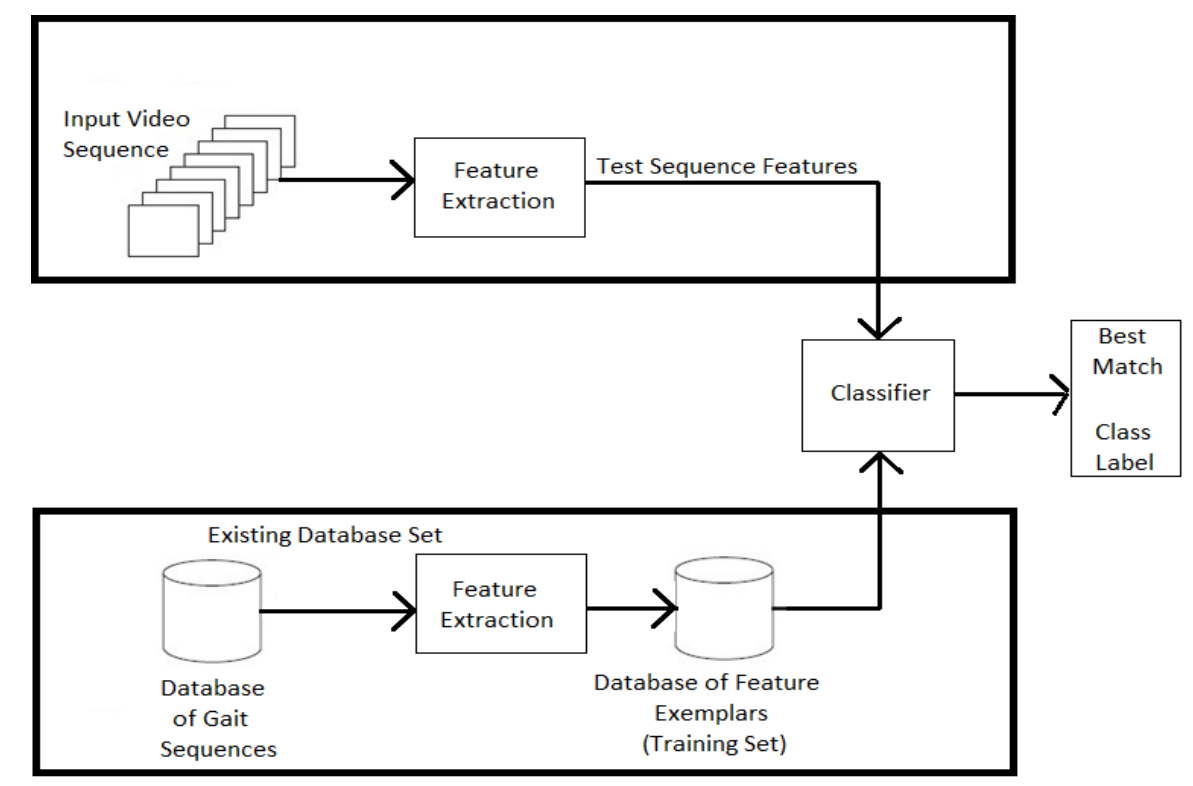

Figure 1: Generalized framework of Gait Recognition 


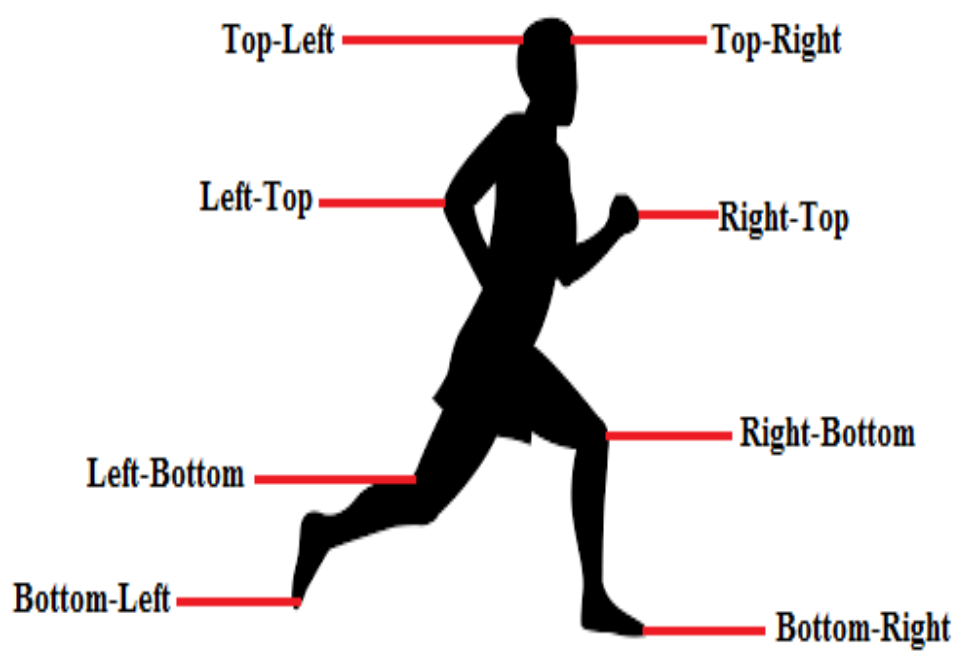

Figure 2: Different Extrema Points for Silhouettes

The step length of the person is calculated by estimating extrema points of the silhouette of the person. Extreme points of the silhouette are shown in figure 2. We have used two extrema points for calculating the step distance which is bottom left and bottom right. These points are found by scanning each and every pixel from the bottom and right side of the frame. As we move upward and sideward while scanning first black pixel we get from the bottom as well as right side, will define the bottom rightmost extreme point of the silhouette. Similarly, we get the bottom leftmost extreme point. These points are mentioned in figure and distance between point $A\left(x_{1}, y_{1}\right)$ and point $B\left(x_{2}, y_{2}\right)$ is the approximated step size, calculated from the following equation of Euclidean distance:

$$
\mathrm{D}=\sqrt{(x 1-x 2)^{2}+(y 1-y 2)^{2}}
$$

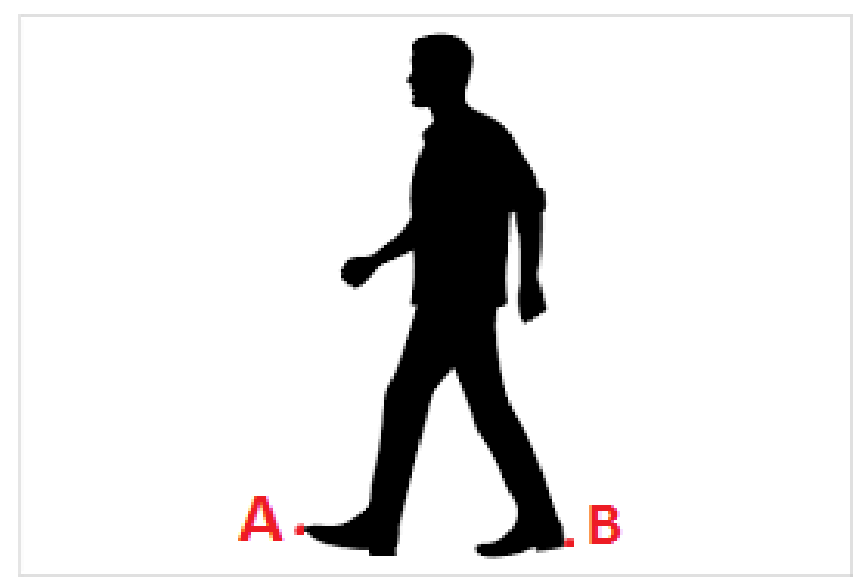

Figure 3: Bottom most extreme points for finding step length 
The point A and B are the extreme points that we get using this function. Then using the Euclidean formula; we get the distance between the points. Thus find the step length of the person.

\subsection{Classification}

An Artificial Neural Network (ANN) [8] [9] is classification system, which mimic the human brain. It uses the phenomenon of the human brain to process information. The system composed of three types of layers with nodes into it. The first layer of the system is input layer, consist of input nodes. The last stage of system is output layer, consist of output nodes. Furthermore, the intermediate layer includes hidden nodes, which are used to map input of output nodes non-linearly. In pattern recognition problems, neural network classifies inputs into a set of target categories. Performance criteria of the neural network are cross-entropy and confusion matrices. The number of the input node of the proposed neural network is 1 and output nodes are 4 .

\section{Experimental Evaluation}

The proposed approach was performed on $2.60 \mathrm{GHz}$ Intel i7 processor of a 64-bit operating system having RAM of $8 \mathrm{~GB}$, MATLAB version 2014a.

\section{Data Set}

CASIA Gait Database [9] has been used in this system as benchmark dataset. In this dataset, we are using multi-view gait database. There are 20 subjects, and the gait data is captured from eleven different views. The data set contains eleven different views but for finding the step length we have used a single view (i.e. the left side view). Here, we are also considering three different variations which are named as clothing, view angle, carrying condition change. Besides the video file, we are also providing silhouettes taken from the video.
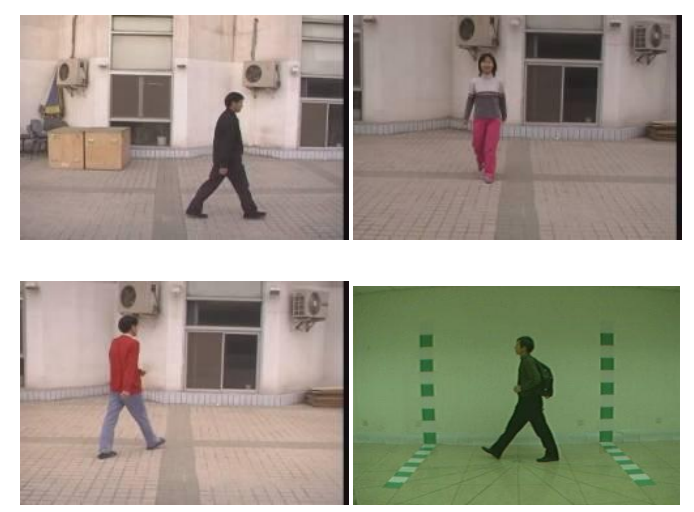

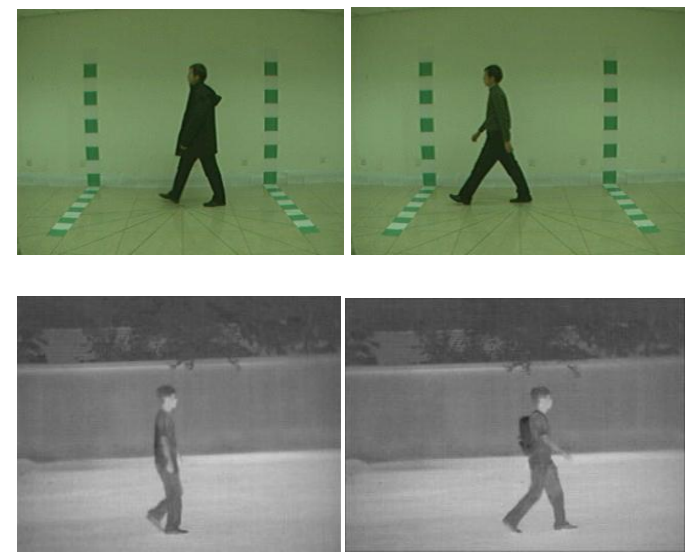

Figure. 4: Snapshots of the data set

The color pictures are converted into binary silhouettes so that the change of color and texture of the cloth doesn't interfere with the recognition system.

The whole data set is divided into 3 categories; Training, testing, and validation. $70 \%$ of dataset is used for training purpose and $15 \%$ is used as the training and remaining $15 \%$ is for validation which is adjusting the parameter of the neural network. The number of hidden layers is 7.

\section{Results}

Table 1 shows the step lengths of ten different people in three consecutive frames. As we can see, the step length of the majority of the people varies from each other, thus it can be used as a distinct feature for identification of the person.

Figure 6 Interprets accuracy of the neural network with proposed feature extraction technique with respect to a various number of hidden layers. The graph shows that we get maximum accuracy at seven hidden layers. The reason might be, at a lower number of hidden layers the input and output mapping is not done appropriately whereas, at a higher number of hidden layer, the network is saturated. Hence we get maximum accuracy by using seven hidden layers in between input layer and the output layer.

The confusion matrix for the proposed approach using combination of step size and neural network has been shown in figure 5. The proposed technique achieved 70\% accuracy. The person 4, person 5, persosn 8 , person 9 , and person 10 are misclassified due to less difference between their step sizes. 


\begin{tabular}{|l|l|l|l|}
\hline Person & D F1 & D F2 & D F \\
\hline 1 & 133.3041635 & 133.3041635 & 129.5569373 \\
\hline 2 & 161.3753389 & 162.1141573 & 161.3753389 \\
\hline 3 & 82.13403679 & 81.46778504 & 82.13403679 \\
\hline 4 & 95 & 95 & 129.4179277 \\
\hline 5 & 133.0037593 & 138.6217876 & 115.3126186 \\
\hline 6 & 174 & 154.175225 & 134.5362405 \\
\hline 7 & 230.2889489 & 228.790297 & 239.1066708 \\
\hline 8 & 113.7013632 & 113.7013632 & 117.5159564 \\
\hline 9 & 127.2831489 & 127.2831489 & 127.2831489 \\
\hline 10 & 97.41663102 & 99.04544412 & 97.65244493 \\
\hline
\end{tabular}

TABLE 1: Step Lengths of Different People

\section{Conclusion}

Due to high-security concerns the recent technologies are evaluating various types of biometrics system. The gait recognition system is one of the up roaring biometrics technology which has gained maximum interest. The proposed technique is simple and effective as step size is a prominent feature of gait. The proposed gait recognition system gives appropriate $70 \%$ accuracy. In future, accuracy can be enhanced by incorporating various features and by integrating another classifier in the system like support vector machines. 


\begin{tabular}{|c|c|c|c|c|c|c|c|c|c|c|c|}
\hline & \multicolumn{11}{|c|}{ Confusion Matrix } \\
\hline 1 & $\begin{array}{c}\mathbf{0} \\
0.0 \%\end{array}$ & $\begin{array}{c}\mathbf{0} \\
0.0 \%\end{array}$ & $\begin{array}{c}0 \\
0.0 \%\end{array}$ & $\stackrel{\mathbf{0}}{0.0 \%}$ & $\begin{array}{c}\mathbf{0} \\
0.0 \%\end{array}$ & $\begin{array}{c}\mathbf{0} \\
0.0 \%\end{array}$ & $\begin{array}{c}\mathbf{0} \\
0.0 \%\end{array}$ & $\begin{array}{c}\mathbf{0} \\
0.0 \%\end{array}$ & $\stackrel{0}{0} \%$ & $\stackrel{0}{0} \%$ & $\begin{array}{l}\text { NaN\% } \\
\text { NaN\% }\end{array}$ \\
\hline 2 & $\begin{array}{c}0 \\
0.0 \%\end{array}$ & $\begin{array}{c}3 \\
10.0 \%\end{array}$ & $\begin{array}{c}0 \\
0.0 \%\end{array}$ & $\begin{array}{c}0 \\
0.0 \%\end{array}$ & $\begin{array}{c}\mathbf{0} \\
0.0 \%\end{array}$ & $\begin{array}{c}\mathbf{0} \\
0.0 \%\end{array}$ & $\begin{array}{c}\mathbf{0} \\
0.0 \%\end{array}$ & $\begin{array}{c}\mathbf{0} \\
0.0 \%\end{array}$ & $\begin{array}{c}0 \\
0.0 \%\end{array}$ & $\begin{array}{c}\mathbf{0} \\
0.0 \%\end{array}$ & $\begin{array}{l}100 \% \\
0.0 \%\end{array}$ \\
\hline 3 & $\begin{array}{c}\mathbf{0} \\
0.0 \%\end{array}$ & $\begin{array}{c}\mathbf{0} \\
0.0 \%\end{array}$ & $\begin{array}{c}3 \\
10.0 \%\end{array}$ & $\begin{array}{c}\mathbf{0} \\
0.0 \%\end{array}$ & $\begin{array}{c}\mathbf{0} \\
0.0 \%\end{array}$ & $\stackrel{0}{0} \%$ & $\begin{array}{c}\mathbf{0} \\
0.0 \%\end{array}$ & $\begin{array}{c}\mathbf{0} \\
0.0 \%\end{array}$ & $\stackrel{0}{0}$ & $\begin{array}{c}\mathbf{0} \\
0.0 \%\end{array}$ & $\begin{array}{l}100 \% \\
0.0 \%\end{array}$ \\
\hline 4 & $\begin{array}{c}\mathbf{0} \\
0.0 \%\end{array}$ & $\begin{array}{c}\mathbf{0} \\
0.0 \%\end{array}$ & $\begin{array}{c}\mathbf{0} \\
0.0 \%\end{array}$ & $\begin{array}{c}2 \\
6.7 \%\end{array}$ & $\begin{array}{c}\mathbf{0} \\
0.0 \%\end{array}$ & $\begin{array}{c}\mathbf{0} \\
0.0 \%\end{array}$ & $\begin{array}{c}\mathbf{0} \\
0.0 \%\end{array}$ & $\begin{array}{c}\mathbf{0} \\
0.0 \%\end{array}$ & $\begin{array}{c}\mathbf{0} \\
0.0 \%\end{array}$ & $\begin{array}{c}3 \\
10.0 \%\end{array}$ & $\begin{array}{l}40.0 \% \\
60.0 \%\end{array}$ \\
\hline 5 & $\begin{array}{c}2 \\
67 \%\end{array}$ & $\stackrel{0}{0}$ & $\begin{array}{c}\mathbf{0} \\
0.0 \%\end{array}$ & $\begin{array}{c}\mathbf{0} \\
0.0 \%\end{array}$ & $\begin{array}{l}2 \\
6.7 \%\end{array}$ & $\begin{array}{c}1 \\
3.3 \%\end{array}$ & $\begin{array}{c}\mathbf{0} \\
0.0 \%\end{array}$ & $\begin{array}{c}\mathbf{0} \\
0.0 \%\end{array}$ & $\begin{array}{c}\mathbf{0} \\
0.0 \%\end{array}$ & $\begin{array}{c}\mathbf{0} \\
0.0 \%\end{array}$ & $\begin{array}{l}40.0 \% \\
60.0 \%\end{array}$ \\
\hline 6 & $\begin{array}{c}0 \\
0.0 \%\end{array}$ & $\begin{array}{c}\mathbf{0} \\
0.0 \%\end{array}$ & $\begin{array}{c}\mathbf{0} \\
0.0 \%\end{array}$ & $\begin{array}{c}\mathbf{0} \\
0.0 \%\end{array}$ & $\begin{array}{c}\mathbf{0} \\
0.0 \%\end{array}$ & $\begin{array}{c}2 \\
6.7 \%\end{array}$ & $\begin{array}{c}\mathbf{0} \\
0.0 \%\end{array}$ & $\begin{array}{c}\mathbf{0} \\
0.0 \%\end{array}$ & $\begin{array}{c}\mathbf{0} \\
0.0 \%\end{array}$ & $\begin{array}{c}\mathbf{0} \\
0.0 \%\end{array}$ & $\begin{array}{l}100 \% \\
00 \%\end{array}$ \\
\hline 7 & $\begin{array}{c}\mathbf{0} \\
0.0 \%\end{array}$ & $\begin{array}{c}\mathbf{0} \\
0.0 \%\end{array}$ & $\begin{array}{c}0 \\
0.0 \%\end{array}$ & $\begin{array}{c}\mathbf{0} \\
0.0 \%\end{array}$ & $\begin{array}{c}\mathbf{0} \\
0.0 \%\end{array}$ & $\begin{array}{c}\mathbf{0} \\
0.0 \%\end{array}$ & $\stackrel{3}{3} \%$ & $\begin{array}{c}0 \\
0.0 \%\end{array}$ & $\begin{array}{c}\mathbf{0} \\
0.0 \%\end{array}$ & $\begin{array}{c}\mathbf{0} \\
0.0 \%\end{array}$ & $\begin{array}{l}100 \% \\
0.0 \%\end{array}$ \\
\hline 8 & $\begin{array}{c}\mathbf{0} \\
0.0 \%\end{array}$ & $\begin{array}{c}\mathbf{0} \\
0.0 \%\end{array}$ & $\begin{array}{c}\mathbf{0} \\
0.0 \%\end{array}$ & $\begin{array}{c}\mathbf{0} \\
0.0 \%\end{array}$ & $\begin{array}{c}1 \\
3.3 \%\end{array}$ & $\begin{array}{c}\mathbf{0} \\
0.0 \%\end{array}$ & $\begin{array}{c}\mathbf{0} \\
0.0 \%\end{array}$ & $\begin{array}{c}3 \\
10.0 \%\end{array}$ & $\begin{array}{c}\mathbf{0} \\
0.0 \%\end{array}$ & $\begin{array}{c}\mathbf{0} \\
0.0 \%\end{array}$ & $\begin{array}{l}75.0 \% \\
25.0 \%\end{array}$ \\
\hline 9 & $\begin{array}{c}\mathbf{1} \\
3.3 \%\end{array}$ & $\begin{array}{c}\mathbf{0} \\
0.0 \%\end{array}$ & $\begin{array}{c}0 \\
0.0 \%\end{array}$ & $\begin{array}{c}1 \\
3.3 \%\end{array}$ & $\begin{array}{c}\mathbf{0} \\
0.0 \%\end{array}$ & $\begin{array}{c}\mathbf{0} \\
0.0 \%\end{array}$ & $\begin{array}{c}\mathbf{0} \\
0.0 \%\end{array}$ & $\stackrel{\mathbf{0}}{0.0 \%}$ & $\begin{array}{c}3 \\
10.0 \%\end{array}$ & $\begin{array}{c}\mathbf{0} \\
0.0 \%\end{array}$ & $\begin{array}{l}60.0 \% \\
40.0 \%\end{array}$ \\
\hline 10 & $\begin{array}{c}\mathbf{0} \\
0.0 \%\end{array}$ & $\begin{array}{c}\mathbf{0} \\
0.0 \%\end{array}$ & $\begin{array}{c}\mathbf{0} \\
0.0 \%\end{array}$ & $\begin{array}{c}\mathbf{0} \\
0.0 \%\end{array}$ & $\begin{array}{c}\mathbf{0} \\
0.0 \%\end{array}$ & $\begin{array}{c}\mathbf{0} \\
0.0 \%\end{array}$ & $\stackrel{\mathbf{0}}{0.0 \%}$ & $\begin{array}{c}\mathbf{0} \\
0.0 \%\end{array}$ & $\begin{array}{c}\mathbf{0} \\
0.0 \%\end{array}$ & $\begin{array}{c}\mathbf{0} \\
0.0 \%\end{array}$ & $\begin{array}{l}\text { NaN\% } \\
\text { NaN\% }\end{array}$ \\
\hline & $\begin{array}{l}0.0 \% \\
100 \%\end{array}$ & $\begin{array}{l}100 \% \\
0.0 \%\end{array}$ & $\begin{array}{l}100 \% \\
0.0 \%\end{array}$ & $\begin{array}{l}66.7 \% \\
33.3 \%\end{array}$ & $\begin{array}{l}66.7 \% \\
33.3 \%\end{array}$ & $\begin{array}{l}667 \% \\
33.3 \%\end{array}$ & $\begin{array}{l}100 \% \\
0.0 \%\end{array}$ & $\begin{array}{l}100 \% \\
0.0 \%\end{array}$ & $\begin{array}{l}100 \% \\
0.0 \%\end{array}$ & $\begin{array}{l}0.0 \% \\
100 \%\end{array}$ & $\begin{array}{l}70.0 \% \\
30.0 \%\end{array}$ \\
\hline & 1 & 2 & 3 & 4 & & $\begin{array}{l}6 \\
\text { et } C\end{array}$ & 7 & 8 & 9 & 10 & \\
\hline
\end{tabular}

Figure 5: Confusion Matrix of Proposed Approach

\section{Accuracy(\%)}

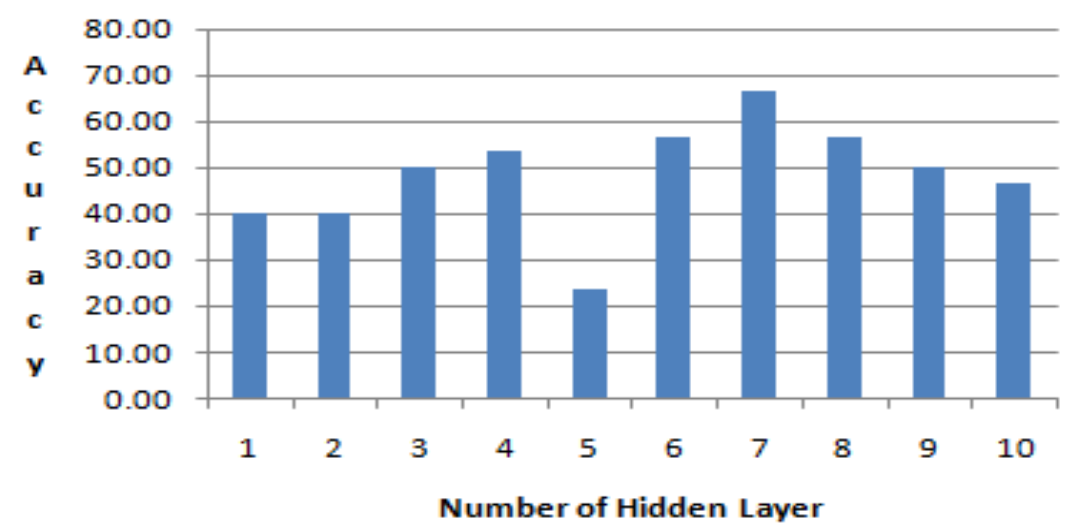

Figure 6: performance of proposed approach accuracy Vs Number of hidden layers 


\section{References}

Cutler, Ross G. "Video-based gait recognition." U.S. Patent 7,330,566, issued February 12, 2008.

Yu Zhong, "Mobile device based gait biometrics ", Winchester, MA (US)

Shroti, Nisha. "A SURVEY PAPER ON HUMAN GAIT RECOGNITION USING PCA \& NEURAL NETWORK." International Journal of Engineering Sciences \& Research Technology 1, no. 4: 743-745.

Wang, Liang, Tieniu Tan, Huazhong Ning, and Weiming Hu. "Silhouette analysis-based gait recognition for human identification." IEEE transactions on pattern analysis and machine intelligence 25, no. 12 (2003): 1505-1518.

Lu, Jiwen, Erhu Zhang, Zhigang Zhang, and Yanxue Xue. "Gait recognition using independent component analysis." In International Symposium on Neural Networks, pp. 183-188. Springer Berlin Heidelberg, 2005.

Muro-de-la-Herran, Alvaro, Begonya Garcia-Zapirain, and Amaia Mendez-Zorrilla. "Gait analysis methods: an overview of wearable and non-wearable systems, highlighting clinical applications." Sensors 14, no. 2 (2014): 3362-3394.

Zhang, Zhaoxiang, Maodi Hu, and Yunhong Wang. "A survey of advances in biometric gait recognition." In Chinese Conference on Biometric Recognition, pp. 150-158. Springer Berlin Heidelberg, 2011.

Patel, Chirag I., Sanjay Garg, Tanish Zaveri, Asim Banerjee, and Ripal Patel. "Human action recognition using fusion of features for unconstrained video sequences." Computers \& Electrical Engineering (2016).

Chirag Patel, Ripal Patel and Palak Patel. Handwritten Character Recognition using Neural Network, International Journal of Scientific and Engineering Research, Volume 2, Issue 4, April 2011.

Zheng, Shuai, Junge Zhang, Kaiqi Huang, Ran He, and Tieniu Tan. "Robust view transformation model for gait recognition." In Image Processing (ICIP), 2011 18th IEEE International Conference on, pp. 2073-2076. IEEE, 2011.

Chirag I Patel and Ripal Patel 'Goal Detection from unsupervised Video Surveillance' in proceeding of ACITY-2011, Chennai, India, and July 2011 published in Springer(LNCS) incommunications in computer and information science (CCIS) series. ISSN:1865-0929

Patel, C. I., and Ripal Patel. "Gaussian mixture model based moving object detection from video sequence." In Proceedings of the International Conference \& Workshop on Emerging Trends in Technology, pp. 698-702. ACM, 2011.

Patel, Chirag I., and Ripal Patel. "Illumination invariant moving object detection." International Journal of Computer and Electrical Engineering 5, no. 1 (2013): 73.

Patel, Chirag I., Sanjay Garg, Tanish Zaveri, and Asim Banerjee. "Top-down and bottom-up cues based moving object detection for varied background video sequences." Advances in Multimedia 2014 (2014): 13.

Vijay Ukani, Sanjay Garg, Chirag I Patel, and Hetali Tank. Efficient Vehicle Detection and Classification for Traffic Surveillance System.International Conference on Advanced Computing and Data Science, Volume: 1, November 2016. 